\title{
Design and Implementation of E-confrontation Simulation System Based on Radar Confrontation Research
}

\author{
Jianwei Yuan ${ }^{1, a}$,Wenpeng $\mathrm{Li}^{2, \mathrm{~b}}$ and Guangjian $\mathrm{Liu}^{3, \mathrm{c}}$ \\ 1,2,3 Luoyang Electronic Equipment Test Centre, Luoyang, China \\ a13383886001@163.com, ${ }^{\mathrm{b}}$ dearlord@live.com, ${ }^{\mathrm{c}} 541008713 @ q q . c o m$
}

Keywords: Electronic countermeasures; Radar confrontation; Simulation system; Design

\begin{abstract}
The development of science and technology makes electronic countermeasures role in modern war is more and more big, the use of Radar reconnaissance enemy and by means of electronic countermeasure jamming enemy Radar ew equipment such as become an indispensable part of modern warfare. In this context, the research on electronic countermeasures and their simulation technology is of great significance. Research and development project, this article is based on electronic countermeasure simulation system in systematically studied the electronic countermeasures simulation research results both at home and abroad, using the experience of others, and carefully analysis the objective requirements under the premise of a set of software was designed and implemented mainly distributed electronic countermeasure simulation system, the hardware is complementary. The system supports the user to design the equipment, scene, and simulation of the electronic countermeasures, and finally evaluate the effectiveness of electronic countermeasures.
\end{abstract}

\section{Introduction}

Electronic against the huge role in modern war, prompted military power to develop electronic countermeasure technology, great effort including research and development of electronic warfare equipment, electronic countermeasure tactics. In this context, the analysis and evaluation of the performance and effectiveness of the weapons and equipment of modern electronic warfare have become the hot points and difficult points in the field of electronic warfare. There is no doubt that practice is the optimal way to evaluate weapon equipment performance, but the practice time consuming, high cost and vulnerable to environmental constraints, not repeat, these shortcomings make drills cannot satisfy to frequent testing electronic warfare equipment performance requirements. Simulation technology is the similarity principle, the basis of the information technology, systems engineering, in computer and related equipment (emulator) as the tool, using the model to study, analysis and evaluation system of multi-disciplinary comprehensive technology. Using modern modeling and simulation technology, build a virtual battlefield environment, the electronic countermeasures simulation, put all kinds of electronic warfare equipment under the same environment simulation, analysis and evaluation, at the same time, this method can be controlled, non destructive, repeatable, distribution in the face of increasingly complex electromagnetic environment, through the study of the electronic warfare simulation means is by far the most effective way.

Research and development project, this article is based on electronic countermeasure simulation system in systematically studied the electronic countermeasures simulation research results both at home and abroad, using the experience of others, and carefully analysis the objective requirements under the premise of a set of software was designed and implemented mainly distributed electronic countermeasure simulation system, the hardware is complementary. This system allows users to design electronic countermeasures equipment, the scene, the scene simulation, and finally evaluate the efficiency of electronic countermeasures, due to the main functions are completed by software simulation, low cost, controllable, repeatable simulation process. 


\section{The Basic Principle of Radar Countermeasures}

The Basic Principle of Radar. The Radar is a device that USES the target's reflection on electromagnetic waves to discover the target and determine its location. Radar working process is as follows: first, the Radar transmitter to airspace launch high-energy electromagnetic wave, electromagnetic wave meet target block will produce certain modulation and scattering, the Radar echo, receive the opportunity to receive after modulation finally after a certain process can signal demodulation of target information.

The Basic Principle of Radar Countermeasures. Radar countermeasures on the basis of Radar technology, the reconnaissance equipment and Radar countermeasures equipment received target Radar electromagnetic signals, on the basis of analysis and calculation of the parameters such as the location of the Radar, frequency, and then according to have prior knowledge of Radar signal processing, concludes that the Radar information such as the function and working condition, finally, the analysis results provide jammer and other related equipment. The basic conditions for Radar countermeasures include: Radar signals to space; The detection receiver receives enough electromagnetic signals; The modulation and parameters of the Radar signal are within the range of the equipment that the parameters are in use. Based on the Radar's detection of target information, the basic methods of Radar countermeasures include: the transmission path of electromagnetic waves that damage Radar detection targets; To generate interference signals into the Radar receiver, to destroy or disrupt the Radar's correct detection of the target signal; Reduce the target's Radar area.

\section{Design and Implementation of Electronic Countermeasures Simulation System based on Radar Countermeasures}

Network Database Design and Implementation. The database is the data center of the whole simulation system, the data center of the database design directly affects the operating efficiency, stability and extensibility of the system. To decrease the complexity of the system and reduce the redundancy of data, in the design process, the number of base table, less as far as possible the number of fields, less as far as possible the number of primary key less as far as possible, shall follow the corresponding national standards and industry standards, all forms to meet the third paradigm. According to actual needs, the project contains two database management system, the corresponding scenarios and evaluate information, scene record list edited scenes in the current system of information; The device list records the basic properties of the supported devices in the system, adding new equipment to the system to access the table; Working mode list records the simulation system to support the operation mode of the signal, signal is added in the scene work mode should conform to a particular job or some combination of work patterns. The basic model list records the mathematical modeling modules needed for the scenario simulation, such as the transmitter, receiver, signal, and so on. The map list, platform list, Radar list, and list of signals are the basic elements of the editing scenario. The electromagnetic distribution table is used to record the important electromagnetic distribution data in real time. The user information table and the device information table records the information about the user and equipment that the design is designed for. Transmitting table, reception table, interference signal object table to record assessment form, in engineering, the assessment will be generated through the data conversion program before the table; The evaluation criteria table records the evaluation criteria used in the evaluation process, and users can access and modify the table by evaluating the criteria. The evaluation achievement table records the reconnaissance scores and the interference scores of the previous assessments, which is the basis of historical performance statistics.

TCP/IP Communication Module Design and Implementation. The most important application of file transfer in this system is to get the evaluation data from the target host. A TCP server is set up on the target host in the system to assess the access to the evaluation data from the TCP server. Evaluation table first issue to get the data to the server application, through the network terminal equipment verify applicant id, and then through the terminal connection data source (one car 
budgeting), access to data from the data source, translates into evaluation system of the available data file is sent to the host evaluation. The user needs to correctly fill in the user name, password, and file path of the target server in the data sharing Settings; Fill in your IP and communication ports in the network connection Settings section; In the server connection Settings area, the server IP address is the IP address of the device terminal, and the upper machine ID is the required parameter for the device terminal connection data source (the spy car).

Apache Server Design. Apache is the world's number one Web server software. The Apache server is open source, and its development team is huge and makes it a cross-platform, portable feature. Select "Editthe Apache HTTPD conf Configuration file" under the Apache installation menu, which means that you can open the Configuration file, and you need to modify the Document Root, which is the site root directory, on line 228. Directory, which is the same address as the Document Root, in line 253; The DirectoryIndex directory index, the name of the home page used, is in line 321. After changing the above path to the system-defined site path, restarting the apche server and accessing the server again, you can see the system login page. The user's login information is processed by the Apache server, and the new or audit operation is completed in the database through the database interface.

The Design and Implementation of the XML Storage Module. Using the Xerces development kit in this system, you need to use XML documents in your project to create a Windows platform for the $\mathrm{C} / \mathrm{C}++$ Xerces development environment. To do this, first download the Xerces development kit, this item is used in the Xerces $\mathrm{C}++2.8 .0$ version, the download is completed, use MD5 verify its integrity, and installed in accordance with the procedure document Xerces -c,; The final step is Build, and xerces-c is an XML development package that can accommodate multiple platforms and multiple development environments, compatible with Windows, Unix, Linx, and more.

In this system, the main role of XML is to store the data files of the scenario data files and the base model. XML is used in conjunction with the database, leveraging its own advantages, greatly simplifying the workload of system development. There are two main approaches to XML document development: the development of an XML parser directly, starting at the bottom; Another is to use the API that the research organization builds on the XML parser. The first method has the advantage of high flexibility, but program development process, process is very complex, the second method is convenient and developers, the benefits of development and high efficiency. A second development method was adopted in this system, using the XML development kit of xercs-c.

$<$ File $>$ BaseModuleSamples $\mid$ SignalSource.dll</File>

$</$ Files $>$

$<$ Description>

$<![$ CDATA[signal source]] $>$

$</$ Description $>$

$</$ BaseModul $>$

The file defines the name of the module, Signal Source, and defines four variables, freq, amp, phase, Sample Freq, and its initial values. The dynamic link library for this module is the signalsour.dll under the Base Module Samples folder. The dynamic link library has functions that implement the module's functionality and provide a specific interface. In the process of scenario simulation, using the Signal Source module, the program will search under Base Module Samples Signal Source. DLL files, after loading the dynamic link library, call its interface, complete the corresponding function. The amplifier is another important module in the simulation system, and a brief amplifier XML document is as follows:

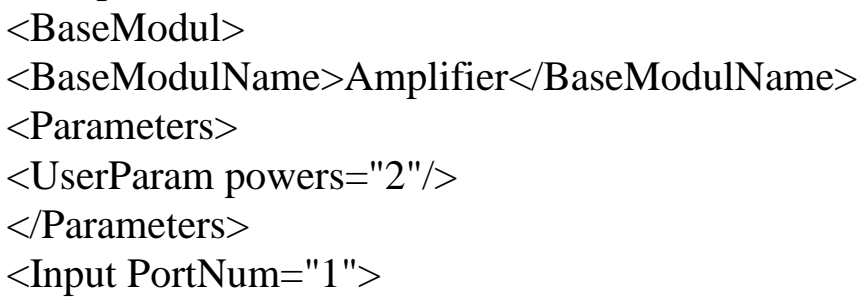




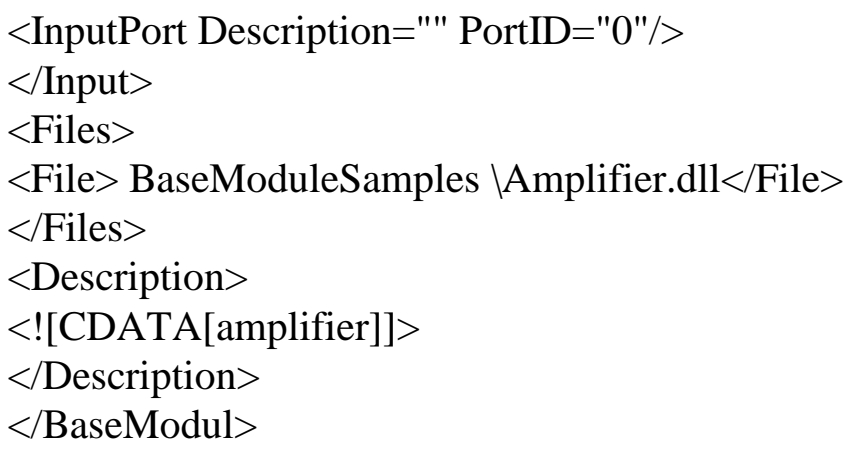

Read the file, the system can get Amplifier module name. The function of the module is to multiply the input and double the power gain. The realization of the module file is Base Module Samples folder Amplifier. DLL. At the start of the simulation, run to invoke the Amplifier, the system to search the Amplifier with Base Module Samples folder. The DLL file, after loading the dynamic link library Amplifier. The DLL, complete the corresponding functions with its function.

$<$ File>BaseModuleSamples $\mid$ SignalSource.dll</File>

$</$ Files $>$

$<$ Description $>$

$<![C D A T A[$ signal source]] $>$

$</$ Description $>$

$</$ BaseModul $>$

The Design and Implementation of the Simulation Module. Radar countermeasures simulation module is the core content of this simulation system, its function is based on a variety of units to establish the mathematical model of real-time calculation of space electromagnetic distribution, and the process of drawing technology simulation Radar confrontation. At the same time, it provides some tools for analyzing the electromagnetic distribution of space, which can be analyzed in the process of simulation. The module can be subdivided multiple sub modules, including the analysis tool of Radar wave disc type hand graph viewer, the viewer, Radar power spectrum diagram viewer, interference figure, such as several sub-modules, the following will be introduced the above-mentioned modules.

Hand graph viewer. Arbitrary point on the selected scenario, setting height of space, and then click the comprehensive situation display menu under the spectrum diagram, frequency spectrum analysis can open the window, as shown in Fig. 1, the upper part of the picture is the map that point of spectrum distribution, the lower part is a waterfall. Can clearly see from the spectrum signal at a frequency distribution, any effect that point signal will leave a mark on the spectrum, spectrum real-time display the current spectrum signal strength, and the waterfall figure recorded history spectrum distribution.

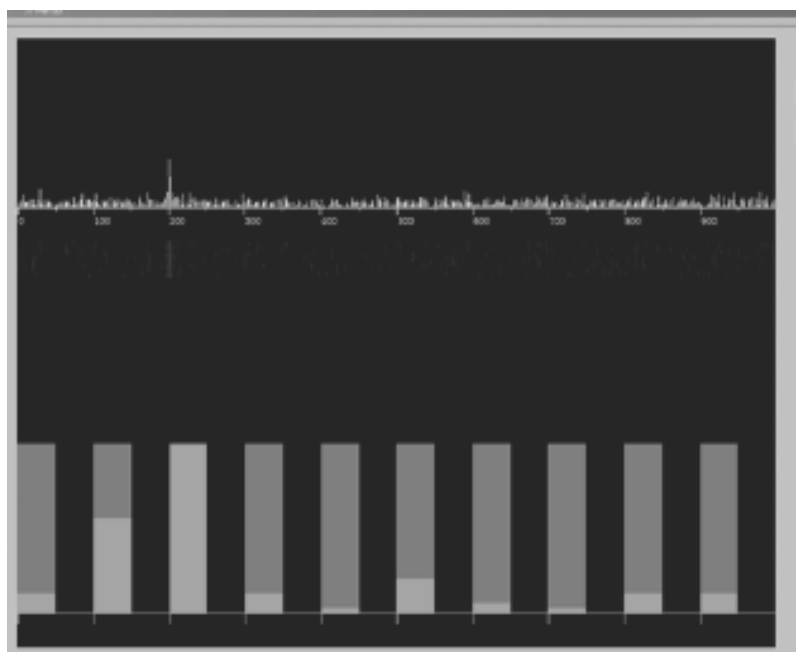

Figure 1. Spectrum 
The power diagram viewer. The power diagram is the coverage of the Radar at a high level. Select the power diagram option below the menu, set a high level, click ok, and you will pop up the power diagram dialog shown in Fig. 2. Radar chart shows the power at a particular level on the level of the Radar beam sectional drawing, LeiDaHuan sweep in the state of present general is a ring, intermediate no Radar signal coverage area called the blind area, Radar can't detect the target in the blind area.

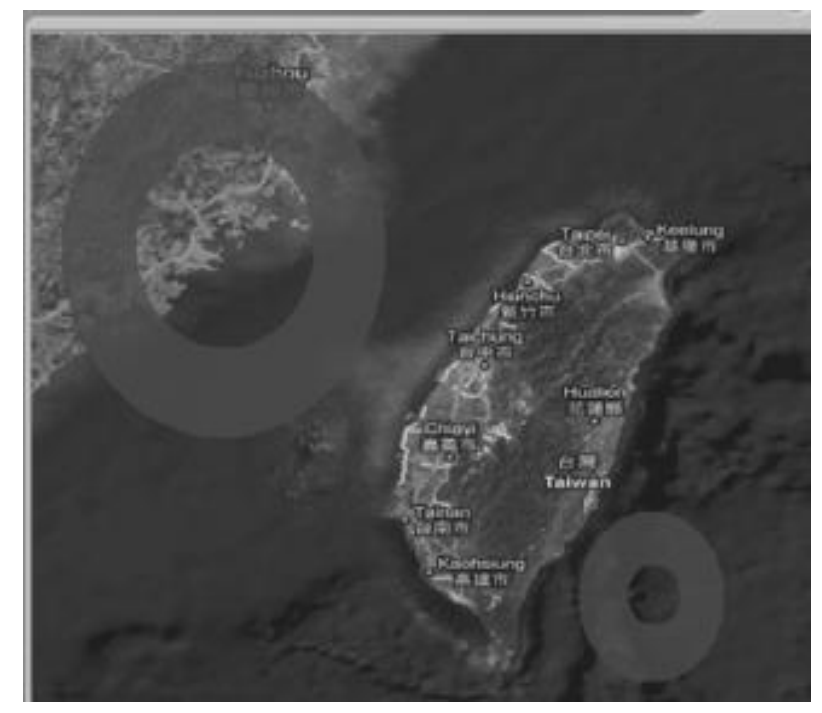

Figure 2. Powerful figure

Hand graph viewer. The Radar beam hand pattern is shown in Fig. 3. The picture is a pitching Angle of 45 degrees of ground Radar, the main lobe of intermediate for the Radar beam, the spindle is the maximum detection range of Radar, can through the mathematical model to calculate the Radar detection range, small lobe called Radar sidelobe, Radar beam main lobe and side lobe in Radar positioning and ranging has important role in the process.

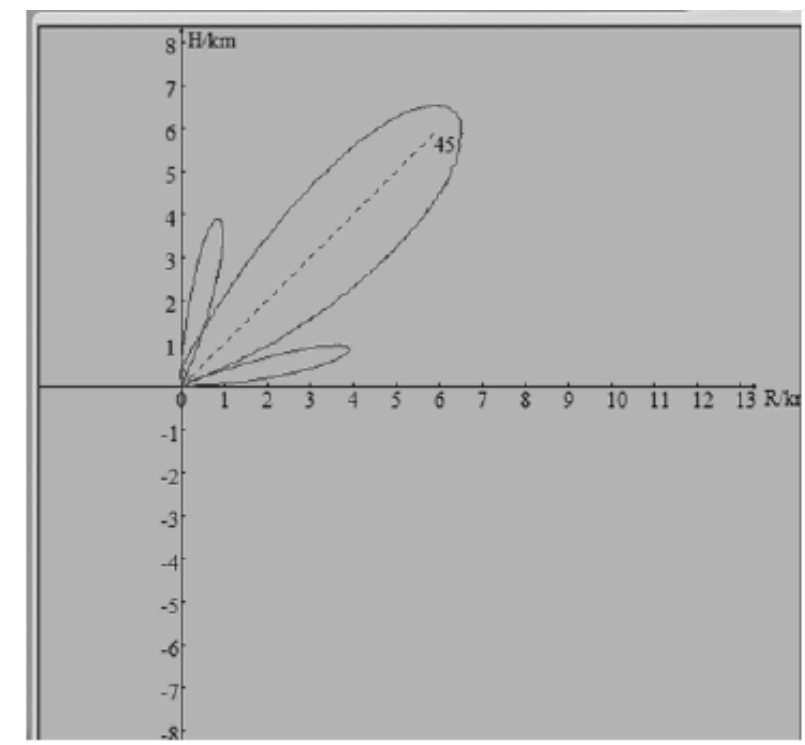

Figure 3. The shape of the hand drawing

Suppress the interference graph viewer. In electronic countermeasures in the process of scene simulation, click the comprehensive situation display menu under suppress interference figure, will pop up as shown in figure 4 to suppress interference figure, center the white areas as Radar detectable area, under normal circumstances, the area should be a circle, but after using the Radar jamming methods for interference, Radar detection range change, under the influence of interference in the north, north of the detection area was significantly suppressed, there is a pit. 


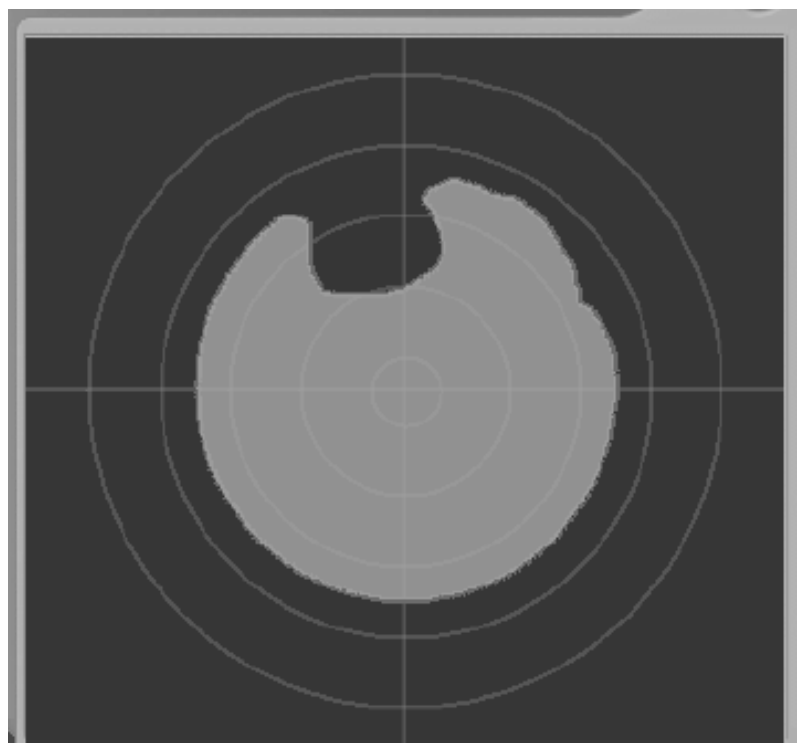

Figure 4. Suppress interference figure

Radar ring map viewer. Integrated situation under the menu LeiDaHuan Ozzie as shown in Fig. 5, which describes the Radar scan and found that the signal process, box in the figure is found and marked the suspicious target Radar.

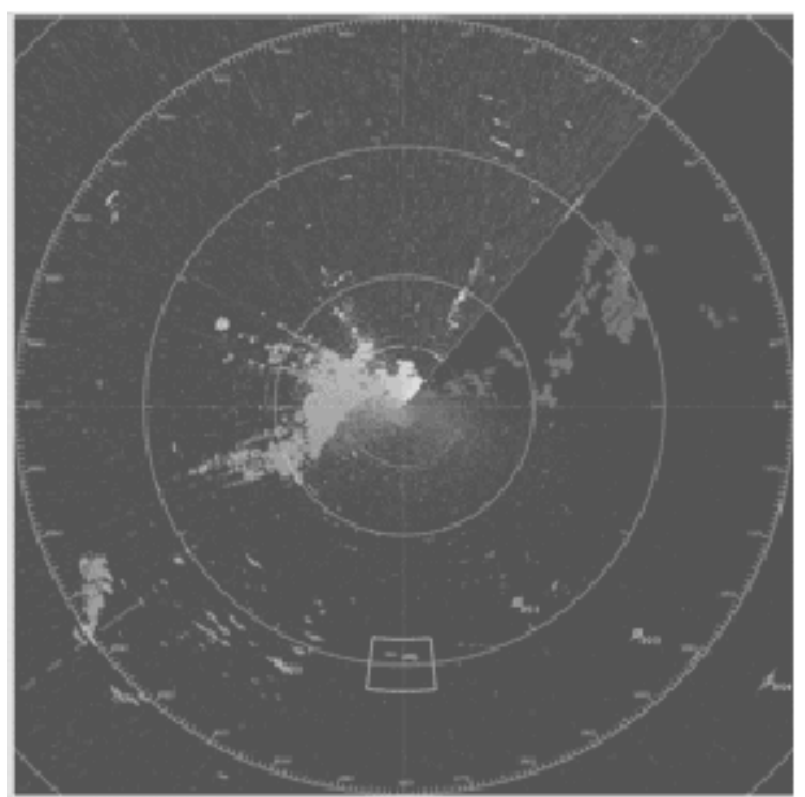

Figure 5. Loop scan of Radar

Evaluate the Design and Implementation of the Module. Assessment software including display and two layers, including display, change volume algorithm, statistical algorithms, databases, training general subject database and $\mathrm{Xml}$ index module, etc. The display layer provides the user with a visible display interface and a performance query interface. The business layer includes the core reading module and the statistical module. Marking module reads the standard answer from scene file and training records, from the Apache server is according to certain change volume algorithm evaluation results, and the evaluation results into the database. Statistics module can call record in the database record, according to the user specified statistical methods of statistics, statistical results will be displayed on the scores query interface, in addition the printing results at according to user requirements. Assessment software business process is: the operator login after evaluating seats, according to the general process can, in turn, selected existing scenario, select training records, save evaluation results, view history, printing history of operation, the system in response to the above message, complete the whole process of the assessment. There are six main objects in the program, and the display interface is responsible for interacting with the user at the front. After the user selects 
the evaluation, the system queries the Xml subject index object for training record information; It then queries the general database for the records of reconnaissance and interference, which are compared in the reading module to complete the rewinding evaluation; The evaluation results are displayed in the display screen, supporting the drawing of the drawing module, printing the document according to the user's requirements, and the performance database on the other. Performance statistics module is another user interaction module, the module on the user can choose a certain statistical method to record history in the database for statistical evaluation results, the statistical results back display interface.

\section{Conclusion}

In this paper, the authors of the current domestic and foreign electronic countermeasures simulation system after a series of research, combined with the requirements of a military unit, the business process of the electronic countermeasure simulation system, module partition, technology has made the certain research and design, finished a set of software in the basis of the supplemented, hardware, software and hardware coordination of electronic countermeasures simulation system, the user use the system makes it easy to design electronic fight scene, exercise training and evaluate the operational effectiveness and, since the system put into use the cooperation unit of recognition and praise.

\section{References}

[1] Li Yunjie, Gao Meiguo, Xie Bingquan. Radar Signal Environment Simulation based on Quadrature Multiple Waveform Synthesis Method[J]. Image and Signal Processing, 2009. CISP '09. 2nd International Congress on.

[2] Susan Symington, Katherine L. Morse, Mikel Petty. IEEE Standard for Modeling and Simulation (M\&S) High Level Architecture(HLA)-Framework and Rules(IEEE Std 1516-2000)[S]. IEEE, IEEE Standard for Modeling and Simulation(M\&S) High Level Architecture(HLA)-Federate Interface Specification[S], Std 1516.1, 2000.

[3] IEEE, IEEE Standard for Modeling and Simulation(M\&S) High Level Architecture(HLA)-Object Model Template(OMT)[S], Std1516.2, 2000.

[4] Mofrad, Reza Fatemi, Sadeghzadeh, R. A. Scenario modeling and simulation for performance prediction of a modern Radar in electronics warfare environment[J]. Radar Symposium (IRS), 2010 11th International. 2010.

[5] Darrel Wright, Clyde Harris, Testing Advanced Distributed Simulation for Use in Electronic Warfare Test and Evaluation[j]. AD-A359369, Jan 1999.

[6] Najgebauer, A., Antkiewicz, R., Tarapata, Z..The autoation of combat decision process in the simulation based perational training support system[J]. Computational Intelligence in Security and Defense Applications, 2007.

[7] Abraham Silberschatz, Henry F. Korth, S. Sudarshan. Database System Concepts, Fourth Editon[M]. Mc Graw Hill Education Co. 2002. 